\title{
Overcoming the barriers to dissemination and implementation of quality measures for gastrointestinal endoscopy: European Society of Gastrointestinal Endoscopy (ESGE) and United European Gastroenterology (UEG) position statement
}

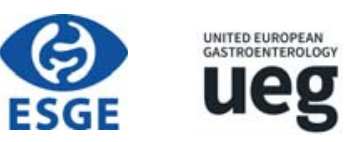

Authors

Raf Bisschops ${ }^{1}$, Matthew D. Rutter ${ }^{2,3}$, Miguel Areia ${ }^{4,5}$, Cristiano Spada6,7, Dirk Domagk ${ }^{8}$, Michel F. Kaminski ${ }^{9}$, Andrew Veitch $^{10}$, Wafaa Khannoussi ${ }^{11,12}$, Ian M. Gralnek ${ }^{13,14}$, Cesare Hassan ${ }^{15}$, Helmut Messmann ${ }^{16}$, Thierry Ponchon ${ }^{17}$, Paul Fockens $^{18}$, Alex Dignass ${ }^{19}$, Mario Dinis-Ribeiro ${ }^{5,20}$

Institutions

1 Department of Gastroenterology and Hepatology, University Hospital Leuven, Leuven, Belgium

2 Department of Gastroenterology, University Hospital of North Tees, Stockton-on-Tees, Cleveland, UK

3 Population Health Sciences Institute, Faculty of Medical Sciences, Newcastle University, UK

4 Gastroenterology Department, Portuguese Oncology Institute, Coimbra, Portugal

5 Center for Health Technology and Services Research (CINTESIS), Faculty of Medicine, University of Porto, Portugal

6 Università Cattolica del Sacro Cuore, Rome, Italy

7 Digestive Endoscopy Unit and Gastroenterology, Fondazione Poliambulanza, Brescia, Italy

8 Department of Medicine I, Josephs-Hospital Warendorf, Academic Teaching Hospital, University of Muenster, Warendorf, Germany

9 Department of Gastroenterological Oncology and Department of Cancer Prevention, The Maria Sklodowska-Curie Memorial Cancer Center and Institute of Oncology, Warsaw, Poland

10 Department of Gastroenterology, Royal Wolverhampton NHS Trust, UK

11 Hepato-gastroenterology department. Mohammed VI University Hospital, Oujda, Morocco

12 Digestive Diseases Research Laboratory (LARMAD), Medical School, Mohammed First University, Oujda, Morocco

13 Ellen and Pinchas Mamber Institute of Gastroenterology and Hepatology, Emek Medical Center, Afula, Israel

14 Rappaport Family Faculty of Medicine, Technion Israel Institute of Technology, Haifa, Israel

15 Endoscopy Unit, Nuovo Regina Margherita Hospital, Rome, Italy

16 Endoscopy Center of the University Clinic of Augsburg, Augsburg, Germany
17 Gastroenterology Division, Hôpital Edouard Herriot, Lyon, France

18 Department of Gastroenterology \& Hepatology, Amsterdam Gastroenterology \& Metabolism, Amsterdam University Medical Centers, University of Amsterdam, The Netherlands

19 Department of Medicine I, Agaplesion Markus Hospital, Goethe University, Frankfurt/Main, Germany

20 Gastroenterology Department, Portuguese Oncology Institute of Porto, Portugal

published online 7.1 .2021

Bibliography

Endoscopy 2021; 53: 196-202

DOI 10.1055/a-1312-6389

ISSN 0013-726X

(c) 2021. European Society of Gastrointestinal Endoscopy

All rights reserved.

This article is published by Thieme.

Georg Thieme Verlag KG, Rüdigerstraße 14,

70469 Stuttgart, Germany

Corresponding author

Raf Bisschops, MD, PhD, Department of Gastroenterology and Hepatology, University Hospital Leuven, KU Leuven,

Herestraat 49, 3000 Leuven, Belgium

Fax: +32-16-344225

raf.bisschops@uzleuven.be

\section{ABSTRACT}

The European Society of Gastrointestinal Endoscopy (ESGE) has developed performance measures and established a framework for quality assessment for gastrointestinal endoscopy in Europe. Most national societies actively undertake initiatives to implement and explicitly endorse these quality indicators. Given this, ESGE proposes that, at a national level, strong leadership should exist to dissemi- 
nate and implement quality parameters. Thus, understanding the potential barriers that may vary locally is of paramount importance. ESGE suggests that each national society should prioritize quality and standards of care in gastro- intestinal endoscopy in their activities and should survey/ understand which measures are a local priority to their members and make measuring quality intrinsic to daily endoscopy practice.

\section{PUBLICATION INFORMATION}

This article is published simultaneously in the journals Endoscopy and the United European Gastroenterology Journal. Copyright 2021 (c) European Society of Gastrointestinal Endoscopy and ( $)$ by the United European Gastroenterology.

$\begin{array}{ll}\text { ABBREVIATIONS } \\ \text { ADR } & \text { adenoma detection rate } \\ \text { ESGE } & \begin{array}{l}\text { European Society of Gastrointestinal Endos- } \\ \text { copy }\end{array} \\ \text { ESGE-TEP ESGE Travelling Endoscopy Programme } \\ \text { GI } & \text { gastrointestinal } \\ \text { JAG } & \text { Joint Advisory Group } \\ \text { NED } & \text { National Endoscopy Database } \\ \text { NHS } & \text { National Health Service (UK) } \\ \text { QIC } & \text { Quality Improvement Committee } \\ \text { SPED } & \text { Portuguese Society of Digestive Endoscopy } \\ \text { UEG } & \text { United European Gastroenterology } \\ \text { UEGW } & \text { UEG week }\end{array}$

\section{Introduction}

Five years ago, the European Society of Gastrointestinal Endoscopy (ESGE) and United European Gastroenterology (UEG) initiated the ESGE quality improvement committee (QIC). The aims of this project were: (1) to improve the global quality of gastrointestinal (GI) endoscopy in Europe and to deliver a patientcentered service in the field of endoscopy; (2) to promote a unifying theme of quality in endoscopy within different activities of the societies; (3) to create a clear quality improvement framework; and (4) to assist all endoscopy units and endoscopists in achieving these standards [1].

From 2015 to 2019, ESGE developed performance measures that allow the assessment of the overall quality of endoscopy in Europe within the various aspects of endoscopy, including the endoscopy service itself [2-11]. During every UEG week (UEGW) and ESGE meeting over the last 4 years, the theme of quality was further addressed, and the work of the different working groups was explained to the members and attendees. Finally, the framework for quality assessment and improvement was established and the bar was set. Now it is time for dissemination and implementation of these quality indicators. There are several barriers preventing the immediate adoption of performance measures in daily routine endoscopy, including lack of motivation, resources, and/or leadership. Such barriers may be successfully overcome by a directed and thoughtful dissemination of the performance measures, as well as by the use of educational or technological interventions.

The aim of this Position Statement by ESGE and UEG is to address those barriers that may prevent acceptance and implementation of performance measures in our endoscopy centers and to suggest possible interventions to overcome such barriers.

\section{Methods}

This Position Statement is based on expert opinion owing to the lack of evidence-based data on the topic of dissemination of performance measures. To address the interest in quality in endoscopy and the possible barriers to implementation, ESGE conducted two surveys among its 49 member societies in 2017, and again in 2019. Responses were received from 32 member societies (27 European and five non-European). This Position Statement addresses both possible barriers to the adoption of performance measures and interventions to overcome these barriers.

\section{Acceptance of quality assessment and barriers to implementation}

\section{RECOMMENDATION}

Endoscopy societies should take a leading role by endorsing, adapting, translating (if deemed necessary), and assisting local health authorities in the implementation of ESGE performance measures.

According to ESGE surveys, $75 \%$ of ESGE member societies explicitly promote performance measures and have actively undertaken initiatives to implement the endoscopy performance measures. There was however a clear discrepancy between promotion and explicit endorsement by member societies. Historically, the interest in quality in endoscopy was driven by the quality of colonoscopy because of its significant effect on patient outcomes [12]. Despite this, the endorsement for lower Gl performance measures in 2019 was only $40 \%$ ( Fig.1). Obviously, endorsement is a process that requires more discussion with individual members and often translation or adaptation to the specific local situation in a country.

We found there is a striking difference between the engagement and promotion of quality measures by national endoscopy societies and the awareness of such measures by national health authorities. In only one-quarter of the countries, are national health authorities aware of the performance measures in endos- 


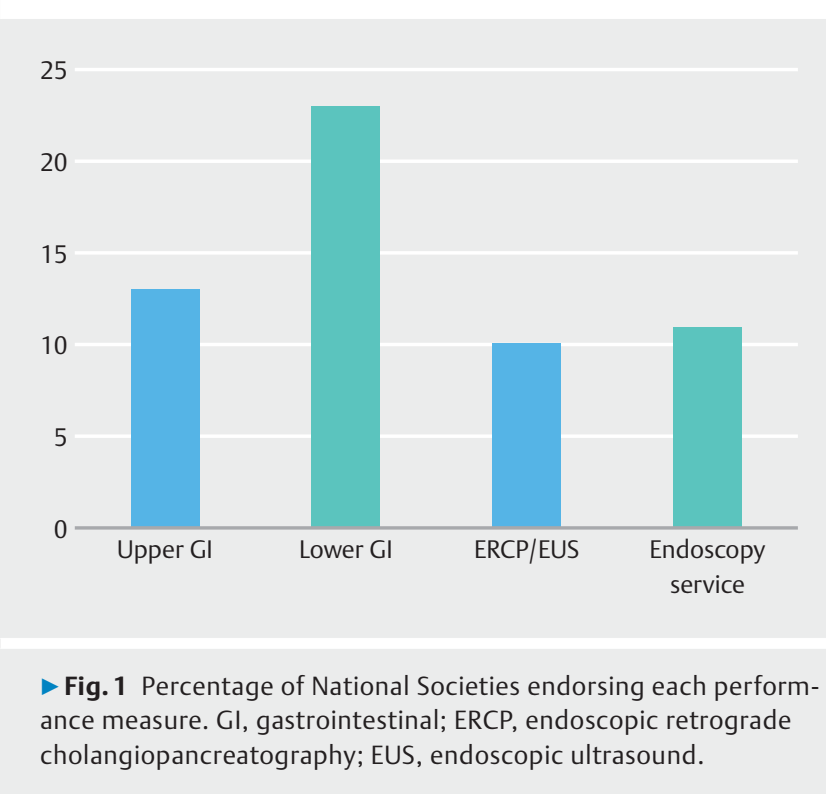

copy and is explicit monitoring of performance required. It can be anticipated however that, in the coming years, national health authorities will become more aware of performance measures and will include them directly into stringent regulations.

\section{Barriers to implementation}

\section{RECOMMENDATION}

Barriers against performance measure implementation should be locally identified and classified into specific categories.

To facilitate dissemination and implementation of endoscopy performance measures, it is important to identify potential barriers. In our surveys, we asked the national societies to identify such possible hurdles. These can be divided into three categories.

\section{Resistance to change}

The first identified barrier concerns the personal motivational level of the endoscopist. In the 2019 ESGE survey, representatives of the national societies reported a lack of enthusiasm and a resistance to change by local practitioners. In addition, some endoscopists feel they are too busy to implement all the performance measures and prefer to continue their endoscopy practice as it is.

"There is nothing more difficult to plan, more doubtful of success, nor more dangerous to manage than the creation of a new system. For the initiators have the enmity of all who would profit by the preservation of the old institutions and merely lukewarm defenders in those who would gain by the new one."
(Machiavelli N. The Prince; 1532. Translated by: Vincent ERP. New York, New York: New American Library; 1952).

Even now, five centuries later, any change in practice will meet resistance.

\section{Lack of/misconceptions of regulation}

A possible reason for practitioner resistance lies in the fact that implementation of performance measures is perceived by many as an administrative burden in an already overly busy daily practice. In addition, there is a fear that implementation of performance measures will come at an additional cost (e.g. computer software packages or payment for administrative support) and that it may prolong endoscopy procedure times. The lack of mandatory regulation by national health authorities and the perceived belief of associated increased costs fails to incentivize endoscopists to speed up the process of quality assessment.

In addition, the adoption of performance measures is generally considered to be an intervention aimed to punish underperforming endoscopists. Instead, performance measure adoption should be considered as a continuous incentive to improve endoscopists' performance by the offer of retraining and other educational interventions.

\section{Practicality of measuring performance measures}

The construct of some of the performance measures is quite complex. To calculate performance measures adequately, inclusion and exclusion criteria need to be considered, or one has to count and indicate how many pictures were taken and what the location was. For instance, accurate photodocumentation of anatomical landmarks and abnormal endoscopic findings is a key performance measure for upper Gl endoscopy. This seems quite straightforward but, in order to audit this, all pictures and anatomical landmarks must be entered into an electronic report or must be checked image by image [2]. In most endoscopy units, a proper information technology (IT) system to assist quality assessment is lacking. Even if an electronic reporting system is used, these often lack uniformity or standardized terminology and allow free text input that compromises automated performance measure extraction [13].

In the ESGE member society survey, only in $15 \%$ of the countries, did more than $90 \%$ of endoscopy services use an electronic reporting system. In 1 out of 3 countries, this coverage was less than $10 \%$ ( $\triangleright$ Fig. 2 ).

\section{How to overcome these barriers}

\section{Prioritize performance measures within your local society}

RECOMMENDATION

Endoscopy societies should prioritize a set of performance measures, taking into consideration local factors, such as disease prevalence, performance measure relevance, and the feasibility of performance measure implementation. 


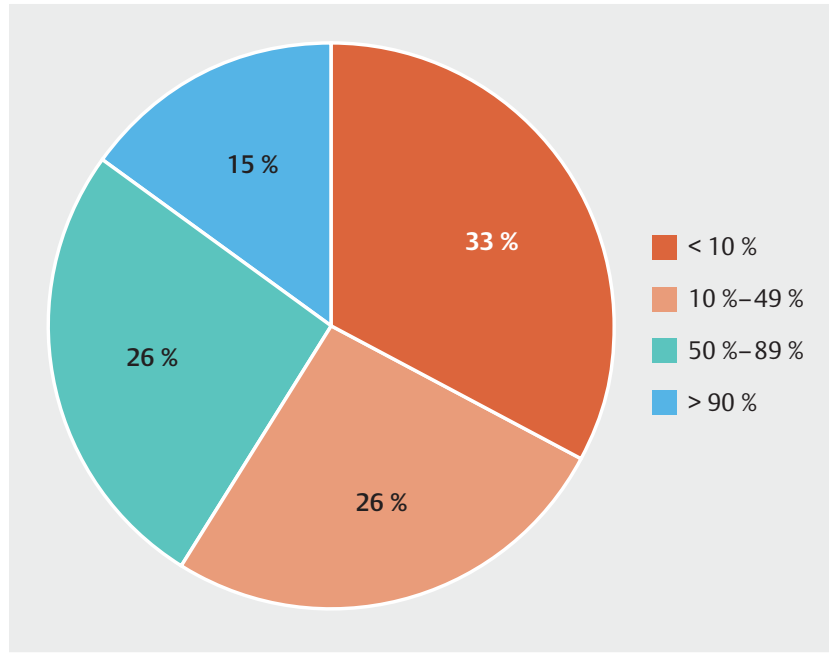

- Fig. 2 Levels of coverage for electronic reporting systems among different countries.

Taking available evidence into consideration, the current set of performance measures are scientifically developed thorough a Delphi process [14]. Every working group tried to reduce the number of performance measures to a minimum to keep the process of auditing realistic and feasible. ESGE wants to emphasize however that, for many performance measures, there is no high-level evidence, and that performance measures may not always be applicable or may be of less importance in a specific country or endoscopy service. For instance, performance measures relating to Barrett's esophagus may be more important in Western Europe, whereas follow-up of gastric intestinal metaplasia may be of more importance in Southern and Eastern Europe. Therefore, ESGE encourages national societies to initiate discussion with their members to select those performance measures that are perceived to be most locally important and/ or relevant.

As an example, the ESGE survey assessed the priorities of member societies with regard to the 11 performance measures for upper Gl endoscopy quality assessment [2]. Surprisingly, three minor performance measures that were classified as possibly less important/more challenging by the upper GI QIC made it into the top six ( $\vee$ Table 1$)$. ESGE strongly encourages national societies to perform a similar survey for all performance measures among their members. This could be done at a national meeting using an online voting system during a session dedicated to quality in endoscopy. For example, the Belgian Society of Gastrointestinal Endoscopy did this at their annual meeting in 2019. By explaining the different performance measures and the reasons or evidence behind them, the national societies can play an essential role in overcoming the resistance to change and gaining acceptance of the concept of measuring quality in $\mathrm{Gl}$ endoscopy.
- Table 1 Top six priorities for upper gastrointestinal quality indicators according to the 2019 ESGE member society survey.

\begin{tabular}{|c|c|c|}
\hline & Performance measure* & Quality indicator* \\
\hline 1 & KPM 4 & $\begin{array}{l}\text { Appropriate use of standardized } \\
\text { terminology }\end{array}$ \\
\hline 2 & KPM 3 & Accurate photodocumentation \\
\hline 3 & KPM 6 & $\begin{array}{l}\text { Follow-up of complications after } \\
\text { therapeutic interventions }\end{array}$ \\
\hline 4 & MPM 10 & $\begin{array}{l}\text { Adequate biopsies according to } \\
\text { MAPS guidelines }\end{array}$ \\
\hline 5 & MPM 7 & Inspection time in the stomach \\
\hline 6 & MPM 8 & $\begin{array}{l}\text { Inspection time in Barrett's } \\
\text { esophagus }\end{array}$ \\
\hline
\end{tabular}

KPM, key performance measure; MPM, minor performance measure; MAPS, Management of precancerous conditions and lesions in the stomach.

*According to Bisschops R et al. [2].

\section{Disseminate the performance measures through your national society}

\section{RECOMMENDATION}

Educational and scientific interventions should be implemented or endorsed by Endoscopy Societies to disseminate performance measures. The use of new or existing electronic databases to audit performance measures, such as the ESGE Quality Check App, is also desirable to facilitate performance measure assessment.

Thus far, many national societies have undertaken initiatives for dissemination and implementation of performance measures, and the evidence below shows that this pays off and improves the overall quality of endoscopy. In an optimal scenario, there is a three-way synergy between national societies, national health authorities, and individual members. However, because the health authorities in many countries are not aware of the quality in endoscopy paradigm, this synergy is often lacking. Nonetheless, there are numerous examples where national societies have taken the lead.

The role of the national societies is in fact two-fold: first, they raise the awareness of quality in endoscopy by assessing problems or lack of quality, and by running individual projects, and thereby they subsequently improve quality; second, this often leads to publications that further substantiate the evidence for certain performance measures. Many quality improvement initiatives are currently undertaken for promoting awareness of quality in endoscopy through dedicated sessions or meetings organized nationally.

For instance, in Italy, a wide variation in cecal intubation rate and adenoma detection rate (ADR) was identified and it was found that, in many instances, split-dose bowel preparation was not used. A subsequent Italian randomized controlled trial 
was conducted and showed a clear increase in ADR when splitdose bowel preparation was used [15]. Direct involvement of different endoscopy services in the identification of a problem and subsequently provision of the evidence that simple measures do actually improve quality should convince even those endoscopists most resistant to change.

In Russia, two projects were initiated for dissemination of the upper GI and lower GI performance measures, STANDUP and QUACOL. They first assessed the quality of colonoscopy and found it to be suboptimal [16]. By organizing more than 50 dedicated workshops and 18 educational events all over the country, they significantly improved the ADR from $18 \%$ to $25.8 \%$ and cecal intubation rate from $86 \%$ to $96.2 \%$.

These types of initiatives raise awareness among endoscopists that simple measures that do not cost anything can improve the quality of endoscopy. Most recently, two interesting reports from Spain and Portugal showed a significant improvement in upper $\mathrm{Gl}$ endoscopy performance following simple quality improvement interventions $[17,18]$. They showed a statistically significant improvement in the use of photodocumentation, use of adequate standardized endoscopic terminology, and the application of correct biopsy protocols.

The involvement of local governmental regulatory agencies usually stimulates quality measurement, often because of the possible financial repercussions and consequences if quality standards are not met. Nonetheless, the involvement of national health authorities may also catalyze quality initiatives without being mandatory. For instance, in 2007, the Austrian Society of Gastroenterology and Hepatology initiated the voluntary reporting of a minimum number of colonoscopies and polypectomies per year via an electronic reporting system. They are backed up by the Austrian Federation of Statutory Insurance institutions and Austrian Cancer Aid. Although providing the data means double data entry for the endoscopist and despite being voluntary, there is a high participation rate because the endoscopy units receive a quality label from the Austrian Society of Gastroenterology and Hepatology if they perform well. This has resulted in a significant improvement in quality in colonoscopy over time in Austria [19].

\section{Start measuring quality indicators: the ESGE Quality Check App}

The degree to which auditing and measuring of quality indicators is achieved depends on the level of development/sophistication of the endoscopy report. Three levels of development can be identified ( $\triangleright$ Fig. $\mathbf{3}$ ). As shown in the results of the ESGE survey, there is wide variability in endoscopy procedure reporting.

In an ideal world, a full electronic reporting system with standardized protocols would be available [20]. Such a reporting system would allow automated capture and feedback of performance measures at an endoscopy service and individual level, virtually in real time.

Because of the involvement of national health authorities to regulate quality in endoscopy, the implementation and widespread use of such electronic reporting systems in endoscopy will undoubtedly be accelerated. This is the case with the

\section{3 levels of development}

- Full electronic reporting with standardized protocols

- Automated capture and feedback of performance measures at service and

1 individual level

- Full electronic reporting with standardized protocols

- No automated capture of performance

2 measures

- Non-standardized electronic reporting (free text) or paper reports

- No automated capture of performance

3 measures

Fig. 3 The different levels of development for gastrointestinal endoscopy reporting.

National Health Service (NHS) in the United Kingdom, which introduced bowel cancer screening in 2006, with a strong emphasis on quality monitoring [21]. Recently, the British Society of Gastroenterology, Association of Upper GI Surgeons, and the Association of Coloproctology of Great Britain and Ireland initiated the National Endoscopy Database (NED) project, under the oversight of the Joint Advisory Group (JAG) on GI Endoscopy. They negotiated with different companies to implement and unify standardized endoscopy reporting systems as a prerequisite for inclusion in the project. As a consequence, they now have an electronic reporting system that allows participating endoscopy centers to directly monitor quality and patient outcomes, without double data entry, directly from the patient's endoscopy report [22].

Even without the explicit influence of national health authorities, national gastroenterology/endoscopy societies can achieve similar effects and obtain standardized reporting systems from software companies. For instance, the Portuguese Society of Digestive Endoscopy (SPED) went through a similar process to unify endoscopy reporting in negotiation with software providers. Recently, the Dutch bowel cancer screening program reported how they developed and deployed a quality register to collect uniform data. This was done in cooperation with commercial endoscopy reporting systems and a national histopathology database to extract data from core hospital resources or histology databases without manual interference of the healthcare providers, again avoiding double data entry [23].

However, in most cases, the endoscopist will have an electronic endoscopy reporting system with or without standardized reporting protocols or will still be using paper reports or free-text digital reports. In those cases, automated capture is impossible. Nonetheless, it is possible to audit these services 
as well. Indeed, measuring approximately 300 gastroscopies and 300 colonoscopies per audit would allow for an adequate snapshot of the quality of those procedures ( $95 \%$ confidence interval $0.87-0.93)$. The main challenge that remains is to take all inclusion and exclusion criteria into consideration during an audit, which in fact may be tedious if done manually. In order to facilitate this, ESGE has developed a quality check app that allows retrospective audit of endoscopy procedures by entering consecutive cases. The ESGE Quality Check App can be downloaded and used on all mobile or desktop platforms. It will guide the endoscopist through different questions to take all exclusion criteria into consideration and will provide the appropriate questions in relation to the pathology that is found. It is estimated that it will take 2 minutes per case entry so, with 3-4 days of administrative work per year, hospital management could support quality assessment and provide a quality snapshot of its colonoscopy and gastroscopy services, without any significant financial investment.

\section{The future role of ESGE and UEG}

ESGE will support and provide the ESGE Quality Check App.

Individuals who are interested in obtaining and using the Quality Check App can contact the ESGE secretariat to ask for access (quality@esge.com). In addition, ESGE will continue to encourage and support projects for the dissemination of quality in endoscopy.

Through the ESGE Travelling Endoscopy Programme (ESGETEP), ESGE can provide dedicated sessions on quality in endoscopy within locally organized meetings. The members of the ESGE QIC are ambassadors of quality in endoscopy and are already undertaking personal initiatives within their home countries to implement quality measurement. The ESGE QIC members also keep up to date with emerging evidence of quality in endoscopy, in order to revise the performance measures in the future. In addition, the ESGE QIC will analyze data from the Quality Check App to build a benchmark of quality throughout Europe for other endoscopy centers and to assess performance measures that may turn out to be less relevant.

It will also be important to address quality in GI endoscopy at the European political level. For this purpose, ESGE and UEG will join forces through public advocacy initiatives, e.g. the Public Affairs Committee of UEG and the ESGE Public Advocacy Committee, to prioritize quality in endoscopy and improve patient outcomes throughout Europe.

\section{Conclusion}

Developing quality indicators for GI endoscopy is a work in progress, with new insights that become apparent every year. ESGE and UEG have developed a set of performance measures for all fields of Gl endoscopy. Although there are still hurdles to overcome, many initiatives throughout Europe have facilitated and promoted quality assessment and put the important issue of quality in Gl endoscopy on the map. ESGE strongly recommends the dissemination and implementation of the quality indicators, as well as the monitoring of these indicators at the local level. This will help to provide the best possible GI endoscopy care for our patients throughout Europe.

This quality of care project may very well serve as a framework to identify quality indicators in other areas of digestive health and help to initiate further quality of care projects in the field of digestive health. Such future initiatives to implement quality of care evaluation into daily practice will hopefully improve the overall care of patients with digestive diseases.

\section{Competing interests}

R. Bisschops is supported by the Research Foundation-Flanders (FWO) and has provided consultancy and received speaker's fees and research support from Norgine, Pentax Europe, and Fujifilm (2017 to present). M. Dinis-Ribeiro received research grants from Olympus and Fujifilm (2018 to present). M.F. Kaminski has provided speaking, teaching, and consultancy services to Olympus (2017 to present), and speaking and teaching services to Fujifilm, from whom he also has equipment on loan (2019 to present). C. Spada provided consultancy to Medtronic (2017-2020). M. Areia, A. Dignass D. Domagk, P. Fockens, I.M. Gralnek, C. Hassan, W. Khanoussi, H. Messmann, T. Ponchon, M.D. Rutter and A. Veitch declare that they have no conflict of interest.

\section{References}

[1] Rutter MD, Senore C, Bisschops R et al. The European Society of Gastrointestinal Endoscopy Quality Improvement Initiative: developing performance measures. Endoscopy 2016; 48: 81-89

[2] Bisschops R, Areia M, Coron E et al. Performance measures for upper gastrointestinal endoscopy: a European Society of Gastrointestinal Endoscopy (ESGE) Quality Improvement Initiative. Endoscopy 2016; 48: 843-864

[3] Bisschops R, Areia M, Coron E. Performance measures for upper gastrointestinal endoscopy: A European Society of Gastrointestinal Endoscopy quality improvement initiative. United Eur Gastroenterol J 2016; 4: 629-656

[4] Kaminski MF, Thomas-Gibson S, Bugajski M et al. Performance measures for lower gastrointestinal endoscopy: a European Society of Gastrointestinal Endoscopy (ESGE) Quality Improvement Initiative. Endoscopy 2017; 49: 378-397

[5] Kaminski MF, Thomas-Gibson S, Bugajski M et al. Performance measures for lower gastrointestinal endoscopy: a European Society of Gastrointestinal Endoscopy (ESGE) quality improvement initiative. United Eur Gastroenterol J 2017; 5: 309-334

[6] Domagk D, Oppong K, Aabakken L et al. Performance measures for ERCP and endoscopic ultrasound: a European Society of Gastrointestinal Endoscopy (ESGE) Quality Improvement Initiative. Endoscopy 2018; 50: 1116-1127

[7] Domagk D, Oppong KW, Aabakken L et al. Performance measures for endoscopic retrograde cholangiopancreatography and endoscopic ultrasound: A European Society of Gastrointestinal Endoscopy (ESGE) Quality Improvement Initiative. United Eur Gastroenterol J 2018; 6: 1448-1460

[8] Spada C, McNamara D, Despott EJ et al. Performance measures for small-bowel endoscopy: a European Society of Gastrointestinal Endoscopy (ESGE) Quality Improvement Initiative. Endoscopy 2019; 51: $574-598$ 
[9] Spada C, McNamara D, Despott E] et al. Performance measures for small-bowel endoscopy: A European Society of Gastrointestinal Endoscopy (ESGE) Quality Improvement Initiative. United Eur Gastroenterol J 2019; 7: 614-641

[10] Valori R, Cortas G, de Lange T et al. Performance measures for endoscopy services: A European Society of Gastrointestinal Endoscopy (ESGE) quality improvement initiative. United Eur Gastroenterol J 2019; 7: 21-44

[11] Valori R, Cortas G, de Lange T et al. Performance measures for endoscopy services: a European Society of Gastrointestinal Endoscopy (ESGE) Quality Improvement Initiative. Endoscopy 2018; 50: 1186-1204

[12] Kaminski MF, Regula J, Kraszewska E et al. Quality indicators for colonoscopy and the risk of interval cancer. NEJM 2010; 362: 1795-1803

[13] Bretthauer M, Aabakken L, Dekker E et al. Reporting systems in gastrointestinal endoscopy: Requirements and standards facilitating quality improvement: European Society of Gastrointestinal Endoscopy position statement. United Eur Gastroenterol J 2016; 4: 172176

[14] Linstone HA, Turoff M. The Delphi Method. Techniques and Applications. 2002: Available at (Accessed 19 October 2020): https://web. njit.edu/ turoff/pubs/delphibook/delphibook.pdf

[15] Radaelli F, Paggi S, Hassan C et al. Split-dose preparation for colonoscopy increases adenoma detection rate: a randomised controlled trial in an organised screening programme. Gut 2017; 66: 270-277
[16] Korolev M, Burdyukov M, Bykov M et al. Quality of colonoscopy in Russia: Report of the Quacol (Quality of Colonoscopy) Study. Gastroenterology 2015; 148: S209-S210

[17] Lisboa-Gonçalves P, Libânio D, Marques-Antunes J et al. Quality of reporting in upper gastrointestinal endoscopy: effect of a simple audit intervention. GE Port J Gastroenterol 2018; 26: 24-32

[18] Córdova H, Sánchez-Montes C, Delgado-Guillena PG et al. Quality indicators for esophagogastroduodenoscopy: A comparative study of outcomes after an improvement programme in a tertiary hospital. Gastroenterol Hepatol 2017; 40: 587-594

[19] Waldmann E, GessI I, Sallinger D et al. Trends in quality of screening colonoscopy in Austria. Endoscopy 2016; 48: 1102-1109

[20] Bretthauer M, Aabakken L, Dekker E et al. Requirements and standards facilitating quality improvement for reporting systems in gastrointestinal endoscopy: European Society of Gastrointestinal Endoscopy (ESGE) Position Statement. Endoscopy 2016; 48: 291-294

[21] Koo S, Neilson L], Von Wagner C et al. The NHS Bowel Cancer Screening Program: current perspectives on strategies for improvement. Risk Manag Healthc Policy 2017; 10: 177-187

[22] Lee T], Siau K, Esmaily $S$ et al. Development of a national automated endoscopy database: The United Kingdom National Endoscopy Database (NED). United Eur Gastroenterol J 2019; 7: 798-806

[23] de Neree Tot Babberich MPM, Ledeboer M, van Leerdam ME et al. Dutch Gastrointestinal Endoscopy Audit: automated extraction of colonoscopy data for quality assessment and improvement. Gastrointest Endosc 2020; 92: 154-162.e1 